\title{
O puerpério e os cuidados de enfermagem: uma revisão sistemática
}

\author{
Puerperium and nursing care: a systematic review \\ Puerperio y cuidado de enfermería: una revisión sistemática
}

\begin{abstract}
Resumo
O período puerperal é o momento do ciclo gravídico-puerperal que corresponde à regressão física gravídica e à passagem para o exercício da maternidade. Ele inicia logo após a dequitação da placenta e termina por volta de seis semanas após o parto, período marcado por diversas mudanças corporais e adaptações emocionais, que podem resultar em desafios que comprometem a relação mãe-filho, os cuidados de enfermagem contribui para a melhora deste ciclo. Analisar as evidências científicas sobre a assistência puerperal e os cuidados de enfermagem; caracterizar os artigos levantados. Trata-se de uma revisão integrativa da literatura realizada nas bases de dados LILACS e SCIELO, acessadas pela BVS. Foram inclusos artigos que estiveram disponíveis eletronicamente, na íntegra, no idioma português, no período de 2013 a 2021. As publicações, durante o intervalo analisado, apresentaram-se em maior quantidade no ano de 2013 com 36\% das publicações. Em relação à base de dados o SCIELO, destacou-se com 86\% das publicações, e quanto ao delineamento metodológico, $93 \%$ dos artigos utilizaram a abordagem quantitativa. A partir dos artigos analisados emergiram duas categorias temáticas: perfil dos idosos residentes em instituições de longa permanência e avaliação da capacidade funcional de idosos em instituições de longa permanência. Conhecer, analisar e compreender as características das puérperas o que contribuiu para o aprofundamento na temática e para a reflexão dos principais pontos a serem avaliados quanto se quer uma assistência sistematizada e individualiza, a fim de proporcionar melhor qualidade de vida para essas puérperas.
\end{abstract}

Palavras-chave: Puérpera; Puérpera fragilizada; Tipos de avaliação de enfermagem; Tipos de cuidados de enfermagem; Assistência domiciliar.

\begin{abstract}
The puerperal period is the moment of the pregnancy-puerperal cycle that corresponds to the physical regression in pregnancy and the transition to the exercise of motherhood. It starts right after delivery of the placenta and ends around six weeks after delivery, a period marked by several bodily changes and emotional adaptations, which can result in challenges that compromise the mother-child relationship. Nursing care contributes to improvement of this cycle. Analyze the scientific evidence on puerperal care and nursing care; characterize the articles raised. This is an integrative literature review carried out in LILACS and SCIELO databases, accessed by the VHL. Articles that were available electronically, in full, in Portuguese, in the period from 2013 to 2021 were included. The publications, during the analyzed period, were presented in greater quantity in the year of 2013 with $36 \%$ of publications. Regarding the SCIELO database, it stood out with $86 \%$ of the publications, and regarding the methodological design, 93\% of the articles used the quantitative approach. From the analyzed articles, two thematic categories emerged: profile of elderly people residing in long-stay institutions and evaluation of the functional capacity of elderly people in long-stay institutions. Knowing, analyzing and understanding the characteristics of puerperal women, which contributed to deepening the theme and to reflecting on the main points to be evaluated in terms of systematized and individualized care, in order to provide better quality of life for these mothers.
\end{abstract}

Keywords: Postpartum; Weakened postpartum; Types of nursing assessment; Types of nursing care; Home assistance.

\section{Resumen}

El puerperio es el momento del ciclo embarazo-puerperal que corresponde a la regresión física en el embarazo y la transición al ejercicio de la maternidad. Comienza inmediatamente después del parto de la placenta y finaliza alrededor de las seis semanas después del parto, período marcado por varios cambios corporales y adaptaciones emocionales, que pueden resultar en desafíos que comprometen la relación madre-hijo. Los cuidados de enfermería contribuyen a la mejora de este ciclo. Analizar la evidencia científica sobre cuidados puerperales y cuidados de 
enfermería; caracterizar los artículos planteados. Se trata de una revisión integradora de la literatura realizada en las bases de datos LILACS y SCIELO, a las que accede la BVS. Se incluyeron los artículos que estuvieron disponibles electrónicamente, íntegramente, en portugués, en el período de 2013 a 2021. Las publicaciones, durante el período analizado, se presentaron en mayor cantidad en el año de 2013 con un 36\% de las publicaciones. En cuanto a la base de datos SCIELO, se destacó con el $86 \%$ de las publicaciones, y en cuanto al diseño metodológico, el $93 \%$ de los artículos utilizó el enfoque cuantitativo. De los artículos analizados surgieron dos categorías temáticas: perfil de las personas mayores residentes en instituciones de larga estancia y evaluación de la capacidad funcional de las personas mayores en instituciones de larga estancia. Conocer, analizar y comprender las características de las puérperas, lo que contribuyó a profundizar en el tema y reflexionar sobre los principales puntos a evaluar en términos de atención sistematizada e individualizada, con el fin de brindar una mejor calidad de vida a estas madres. Palabras clave: Posparto; Posparto debilitado; Tipos de evaluación de enfermería; Tipos de cuidados de enfermería; Asistencia domiciliaria.

\section{Introdução}

O período puerperal é o momento do ciclo gravídico-puerperal que corresponde à regressão física gravídica e à passagem para o exercício da maternidade. Ele inicia logo após a dequitação da placenta e termina por volta de seis semanas após o parto, período marcado por diversas mudanças corporais e adaptações emocionais, que podem resultar em desafios que comprometem a relação mãe-filho (Castiglioni et al., 2020).

A mulher vivencia profundas transformações no puerpério, expondo-se à maior frequência de agravos que são causas específicas de morbimortalidade materna. O Ministério da Saúde, valendo-se do Programa de Humanização do Pré-natal e Nascimento (PHPN), recolocou em pauta o acesso e a qualidade do cuidado no ciclo gravídico-puerperal. Como parte da integralidade no Sistema Único de Saúde (SUS), o puerpério é uma das áreas básicas de atuação da Estratégia Saúde da Família (ESF), modelo preferencial da atenção primária à saúde no Brasil (Corrêa et al., 2017).

No puerpério, mulheres, recém-nascidos (RN) e famílias apresentam necessidades de saúde. Apesar de se esperar que seja um período de vivências saudáveis, podem surgir problemas de ordem física, subjetiva, relacional e social. As profundas transformações vivenciadas nesse ciclo podem expor as mulheres a agravos específicos de morbimortalidade materna (Castiglioni et al., 2020).

Atualmente, nota-se a fragmentação do cuidado oferecido à mulher, o que pode prejudicar a qualidade da assistência prestada. Ao longo dos anos, programas e políticas públicas de saúde foram criados a fim de assegurar a assistência de qualidade com foco em sua integralidade, dentre as quais se destacam o Programa de Atenção Integral a Saúde da Mulher (PAISM) em 1983 e a Política Nacional de Atenção Integral a Saúde da Mulher (PNAISM) em 2004 (Silva et al., 2020).

Diante dos benefícios relativos à consulta de enfermagem no puerpério, acredita-se que reconhecer as principais necessidades da mulher nesse período e oferecer assistência sistematizada pode trazer benefícios para o binômio mãe-filho, como a identificação e tratamento precoce das patologias específicas do ciclo (Silva et al., 2020).

Segundo a normatização da Primeira Semana de Saúde Integral, os serviços de atenção primária à saúde devem realizar a visita domiciliar nos primeiros sete dias pós-alta da maternidade para avaliar as condições de saúde, orientar as ações promocionais e preventivas em saúde e identificar situações de riscos e intercorrências para a adoção de condutas adequadas. As ações no puerpério imediato envolvem, principalmente, o trabalho do enfermeiro articulado ao do agente comunitário de saúde (ACS), sendo solicitada avaliação médica quando são detectadas alterações (Brasil, 2006). O Ministério da Saúde preconiza a programação rotineira da visita domiciliar pelas equipes para priorizar indivíduos e famílias de maior risco (Brasil, 2012).

O regresso da mulher e do RN ao serviço de saúde e entre 7 a 10 dias após o parto, deve ser encorajado não somente durante o pré-natal, mas também na maternidade e nas visitas domiciliares pelos agentes comunitários de saúde, visto que a maioria das situações de morbidade e mortalidade materna e neonatal ocorrem na primeira semana após o parto. Os serviços de saúde devem aproveitar a ocasião da consulta ou visita puerperal para promover todo o cuidado previsto para a PSSI (Brasil, 
2013).

\section{Metodologia}

O presente estudo utilizará como método a Revisão Sistemática da Literatura, pois a Revisão, como uma forma de pesquisa, permite revisar, criticar e sintetizar a literatura científica representativa sobre um tópico ou um assunto de forma integrada, gerando novas abordagens e perspectivas sobre o assunto revisado. Essa metodologia representa um dos recursos da Prática Baseada em Evidências (PBE), ou seja, ela possibilita a síntese e a análise do conhecimento científico já produzido sobre o tema investigado (Lacerda et al., 2012; Broome, 1993; Ganong, 1987).

Segundo Carliner (2011), essa forma de revisão, na última década, apresentou uma notável penetração na área do cuidado à saúde baseado em evidência ou prática baseada em evidência, a qual está associado a métodos de pesquisa, ainda que sob diferentes matrizes epistemológicas.

O estudo do tipo revisão sistemática obedecerá às seguintes etapas: 1. Formular uma questão de investigação; 2. Produzir um protocolo de investigação e efetuar o seu registo (itens 1 e de 3 a 8 devem constar no protocolo de elaboração da revisão sistemática); 3. Definir os critérios de inclusão e de exclusão; 4. Desenvolver uma estratégia de pesquisa e pesquisar a literatura - encontrar os estudos; 5. Seleção dos estudos; 6. Avaliação da qualidade dos estudos; 7. Extração dos dados; 8. Síntese dos dados e avaliação da qualidade da evidência e 9. Disseminação dos resultados - Publicação (Donato; Donato, 2019).

Para a estruturação da questão de investigação, adotou-se a estratégia PICO, em que P é o paciente ou o problema, I é a intervenção, C é o controle ou a comparação, e O é o desfecho. Seguiu-se, a partir da questão de pesquisa - que produções científicas existem em se tratando da avaliação da capacidade funcional dos idosos em instituições de longa permanência. A estratégia PICO foi representada da seguinte forma: Paciente (puéperas), Intervenção (avaliação de Enfermagem), Controle ou comparação (não se aplica), Desfecho (cuidados de Enfermagem), de acordo com o Quadro 1.

Quadro 1 - Estratégia PICO formulada a partir da questão de pesquisa.

\begin{tabular}{|l|l|l|}
\hline \multicolumn{2}{|c|}{ ESTRATÉGIA } & DESCRITORES \\
\hline P (Paciente) & Puérperas & $\begin{array}{l}\text { Puérpera } \\
\text { Puérpera Fragilizada }\end{array}$ \\
\hline I (Intervenção) & Avaliação de Enfermagem & Tipos de Avaliação de Enfermagem \\
& & \\
\hline O (Desfecho) & Cuidados de Enfermagem & Tipos de Cuidados de Enfermagem \\
\hline \multicolumn{2}{|c|}{ AND NOT } & Assistência Domiciliar \\
\hline
\end{tabular}

Fonte: BVS.

Para a busca ou a amostragem na literatura, utilizou-se a combinação dos descritores do P (Paciente), os descritores do I (Intervenção) e os descritores do O (Desfecho) somados ao operador booleano AND, OR e NOT, como esquematizado a seguir: (Puérperas OR Puérpera Fragilizada) AND (Avaliação de Enfermagem OR Tipos de Avaliação de Enfermagem) AND (Cuidados de Enfermagem AND NOT (Assistência Domiciliar)).

Os artigos foram selecionados de acordo com critérios de inclusão: artigos indexados nas bases de dados Literatura científica e técnica da América Latina e Caribe (LILACS), Scientific Electronic Library Online (SCIELO) via Biblioteca Virtual em Saúde (BVS), que estiverem disponíveis eletronicamente, na íntegra, no idioma português, no período de 2013 a 2021. Com a finalidade de reduzir possíveis vieses nos resultados da pesquisa, foram excluídos artigos de revisão bibliográfica, de estudo de caso, artigos de opinião, assim como artigos do tipo teórico-reflexivos, por não retratarem resultados de 
investigações, e artigos duplicados nas referidas bases de dados.

A compilação bibliográfica foi realizada por meio de um instrumento já validado de Ursi (2005) (ANEXO), o qual contempla os seguintes itens: identificação do artigo original, características metodológicas do estudo, avaliação do rigor metodológico, das intervenções mensuradas e dos resultados encontrados. Para análise e posterior síntese dos artigos que atenderam aos critérios de inclusão, foram utilizados dois quadros sinópticos especialmente construídos para esse fim, que contemplaram as seguintes variáveis, consideradas pertinentes: ano de publicação, abordagem metodológica, periódicos, número de ordem dos artigos, autor(es), título do artigo, base de dados, objetivos, principais resultados e categoria em que se inseriu cada estudo.

A apresentação dos resultados e da discussão dos dados obtidos foi realizada de forma descritiva, possibilitando ao leitor a avaliação da aplicabilidade da revisão integrativa elaborada, de forma a atingir o objetivo desse método. Para essa apresentação, foram elaboradas categorias temáticas, com o intuito de promover a compreensão dos conteúdos analisados, dividindo-se em cinco etapas, pré-análise, exploração do material e tratamento dos resultados, a inferência e a interpretação, visto que é a melhor forma de análise de material referente à saúde (Minayo,2007).

A etapa da pré-análise compreende a leitura flutuante, a constituição do corpus, a formulação e a reformulação de hipóteses ou de pressupostos. No que se refere à etapa da exploração do material, o investigador busca encontrar categorias que são expressões ou palavras significativas em função das quais o conteúdo de uma fala será organizado. A análise temática tradicional trabalha inicialmente essa fase, recortando o texto em unidades de registro que podem constituir palavras, frases, temas, personagens e acontecimentos, indicados como relevantes para a pré-análise (Minayo, 2007).

Subsequentemente, o pesquisador escolhe as regras de contagem por meio de codificações e de índices quantitativos e, por último, realiza a classificação e a agregação dos dados, escolhendo as categorias teóricas ou empíricas, responsáveis pela especificação do tema. Posteriormente, o pesquisador propõe inferências e realiza interpretações, inter-relacionando-as com o quadro teórico desenhado inicialmente, sugerida pela ela leitura do material (Minayo, 2007).

\section{Resultados e Discussão}

A coleta de dados foi realizada no mês de julho de 2021, e, para a apresentação das etapas de seleção dos artigos, utilizou-se um quadro demonstrativo (Quadro 2).

Quadro 2 - Apresentação da coleta de dados.

\begin{tabular}{|c|c|c|c|c|}
\hline Estratégia de Busca & Base de Dados & $\begin{array}{l}\text { Critérios de } \\
\text { Inclusão }\end{array}$ & $\begin{array}{c}\text { Critérios de } \\
\text { Exclusão }\end{array}$ & Total \\
\hline $\begin{array}{l}\text { (tw:((tw:(Puérpera)) } \\
\text { (tw:(Puérpera } \\
\text { Fragilizada)))) } \\
\text { (tw:((tw:(Avaliação } r \text { de } \\
\text { Enfermagem }) \text { OR } \\
\text { (tw:(Tipos de Avaliação de } \\
\text { Enfermagem)))) AND } \\
\text { (tw:(Tipos de Cuidados de } \\
\text { Enfermagem)) AND NOT } \\
\text { (tw:(Assistência } \\
\text { Domiciliar)) }\end{array}$ & $\begin{array}{l}\text { Total: } 1776 \\
\text { LILACS: } 51 \\
\text { SCIELO: } 1725\end{array}$ & $\begin{array}{l}\text { Disponível: } 39 \\
\text { Português: } 30 \\
\text { Ano: } 21 \\
\text { Puérperas: } 14\end{array}$ & $\begin{array}{l}\text { Tema: } 3 \\
\text { Duplicado: } 1\end{array}$ & $\begin{array}{l}\text { 14-04=10 } \\
\text { LILACS: } 2 \\
\text { SCIELO: } 8\end{array}$ \\
\hline
\end{tabular}

Fonte: Pesquisa direta na BVS via portal CAPES.

A Tabela 1 mostra as informações obtidas, elaborou-se, com os principais dados do estudo, uma tabela de acordo com o proposto na metodologia. Na tabela abaixo foram incluídas algumas variáveis consideravelmente importantes para o estudo. 
Categorização dos estudos científicos de acordo com as variáveis: ano de publicação; abordagem metodológica; e periódicos (n=14), Teresina, PI, 2013 a 2021

Tabela 1 - Categorização das publicações científicas.

\begin{tabular}{|c|c|}
\hline Variáveis & $\mathbf{N}$ \\
\hline \multicolumn{2}{|l|}{ Ano de Publicação } \\
\hline 2013 & 1 \\
\hline 2017 & 1 \\
\hline 2018 & 1 \\
\hline 2019 & 2 \\
\hline 2020 & 4 \\
\hline 2021 & 1 \\
\hline \multicolumn{2}{|l|}{ Base de dados } \\
\hline LILACS & 2 \\
\hline SCIELO & 8 \\
\hline \multicolumn{2}{|l|}{ Abordagem Metodológica } \\
\hline Quantitativa & 9 \\
\hline Caso - controle & 1 \\
\hline \multicolumn{2}{|l|}{ Periódicos } \\
\hline Acta Paulista de Enfermagem & 1 \\
\hline Ciência e Saúde Coletiva & 3 \\
\hline Revista Brasileira de Enfermagem (REBEn) & 3 \\
\hline Revista Gaúcha de Enfermagem & 1 \\
\hline Revista de Saúde Pública & 2 \\
\hline
\end{tabular}

Fonte: Banco de dados BVS.

Foram analisados 10 artigos, publicados na íntegra, na área temática deste estudo, compreendidos entre os anos de 2013 a 2021. As publicações, durante o intervalo analisado, apresentaram-se em maior quantidade no ano de 2020 , com $36 \%$ das publicações, seguido dos anos de 2019, com 21,4\%, do ano de 2013, 2017 e 2018 com o restante. Em relação à base de dados, o SCIELO se destacou com $86 \%$ das publicações em comparação com a base de dados LILACS, que representaram $7 \%$ das publicações. Quanto ao delineamento metodológico, 93\% dos artigos utilizaram a abordagem quantitativa, e 7\% utilizaram caso-controle. 
Tabela 1 - Caracterização dos estudos científicos de acordo com as variáveis: número de ordem, autor(es), título do artigo, base de dados, objetivos, principais resultados dos estudos e categoria (n=14), Teresina, PI, 2011 a 2016.

\begin{tabular}{|c|c|c|c|c|c|c|}
\hline o & Autor (es) & Título do artigo & $\begin{array}{ll}\text { Base } & \text { de } \\
\text { dados }\end{array}$ & Objetivos & Principais resultados & Categoria \\
\hline & \multirow[t]{2}{*}{$\begin{array}{l}\text { Prigor } \\
\text { et al. }\end{array}$} & $\begin{array}{l}\text { O papel do Enfermeiro } \\
\text { no cuidado à puérpera }\end{array}$ & LILACS & $\begin{array}{l}\text { Identificar o papel do } \\
\text { enfermeiro } \\
\text { transição puerperal nos }\end{array}$ & 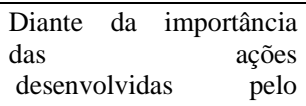 & \\
\hline & & & & $\begin{array}{l}\text { contextos } \\
\text { hospitalar e comunitário }\end{array}$ & $\begin{array}{l}\text { enfermeiro durante o } \\
\text { período puerperal, é } \\
\text { imprescindível que tome } \\
\text { seu espaço de atuação, } \\
\text { para } \\
\text { a assistência se torne } \\
\text { mais qualificada. }\end{array}$ & 1 \\
\hline
\end{tabular}

$\begin{array}{lllll}\text { Ebling et } & \begin{array}{l}\text { Compreensão } \\ \text { cuidado na ótica } \\ \text { puérpera }\end{array} & \text { da } & \text { LILACS } \\ & & \end{array}$

I

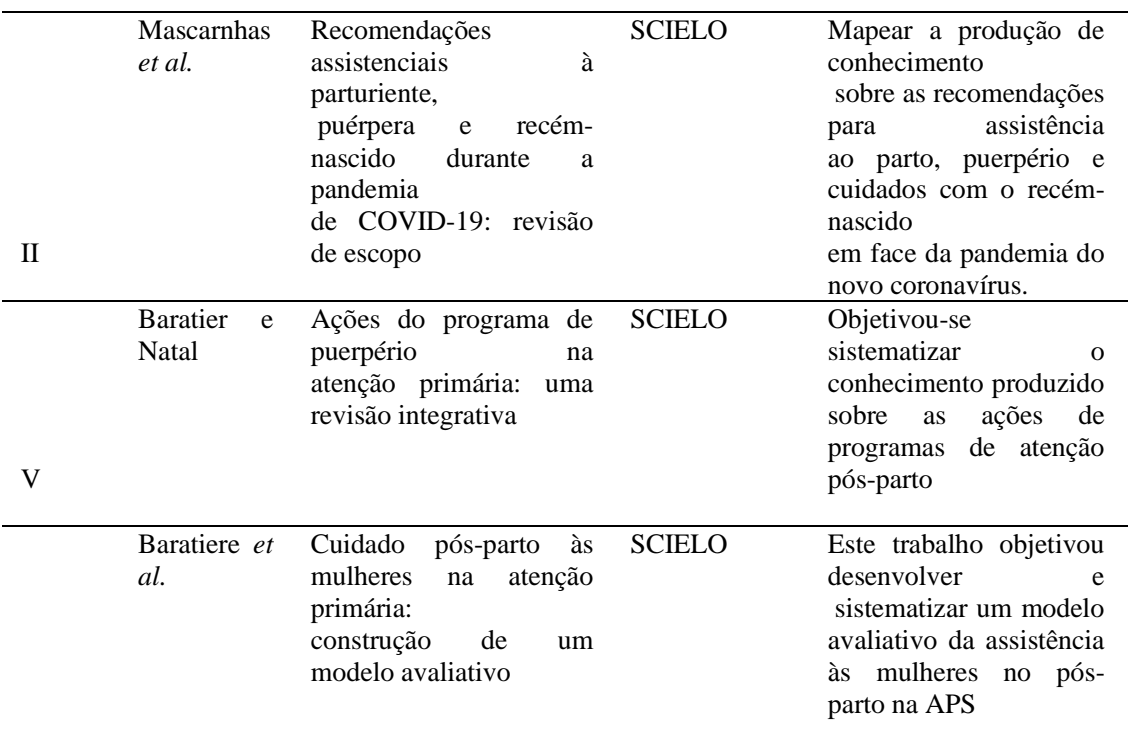

Conhecer a percepção
do cuidado de
enfermagem sob o olhar
de puérperas em uma
maternidade, durante a
permanência em
alojamento conjunto.

Os dados nos levaram à

categoria:

"o cuidado de

enfermagem como

atenção:

avanços, obstáculos e

desafios". Evidenciou-se

um cuidado atencioso,

mas com ênfase nos

procedimentos técnicos e

voltados para o recém-

nascido.

Recomendações sobre a

assistência puerperal.

Os resultados apontam

que: a APS possui

estrutura

física para atenção à

puérpera, porém com

déficit em recursos humanos e materiais

Verificou-se que a

assistência pós-parto

é avaliável por meio de

uma análise de

implantação e que a

teoria do programa

definida pode ser

utilizada por diversos

atores

Felix et al. Atuação da enfermagem

SCIELO

O objetivo deste estudo foi identificar como frente à depressão pósparto nas consultas de puericultura a enfermagem atua

Observamos que os enfermeiros não tinham um conceito para a

doença, mas eram capazes de identificar fatores relacionados à doença. Houve sensibilização o que, até então não acontecia, promovendo subdiagnóstico.

$\begin{array}{lll}\text { Bratiere e } & \begin{array}{l}\text { Ações do programa de } \\ \text { puerpério na atenção } \\ \text { primária: }\end{array}\end{array}$
uma revisão integrativa

II

Giantaglia
et al.

III etal. cuidado em um programa de residência enfermagem obstétrica: possibilidades

SCIELO

SCIELO

\section{Objetivou-se}

sistematizar

conhecimento produzido

sobre

as ações de programas

de atenção pós-parto no âmbito da APS

Descrever as

potencialidades e os desafios das

residentes sobre a

experiência vivenciada
Os resultados apontam

que: a APS possui

estrutura

física para atenção à

puérpera, porém com

déficit em recursos humanos e materiais.

Das reflexões das

residentes, por meio das

categorias emergentes,

pôde-se verificar que

estas reconheciam como 


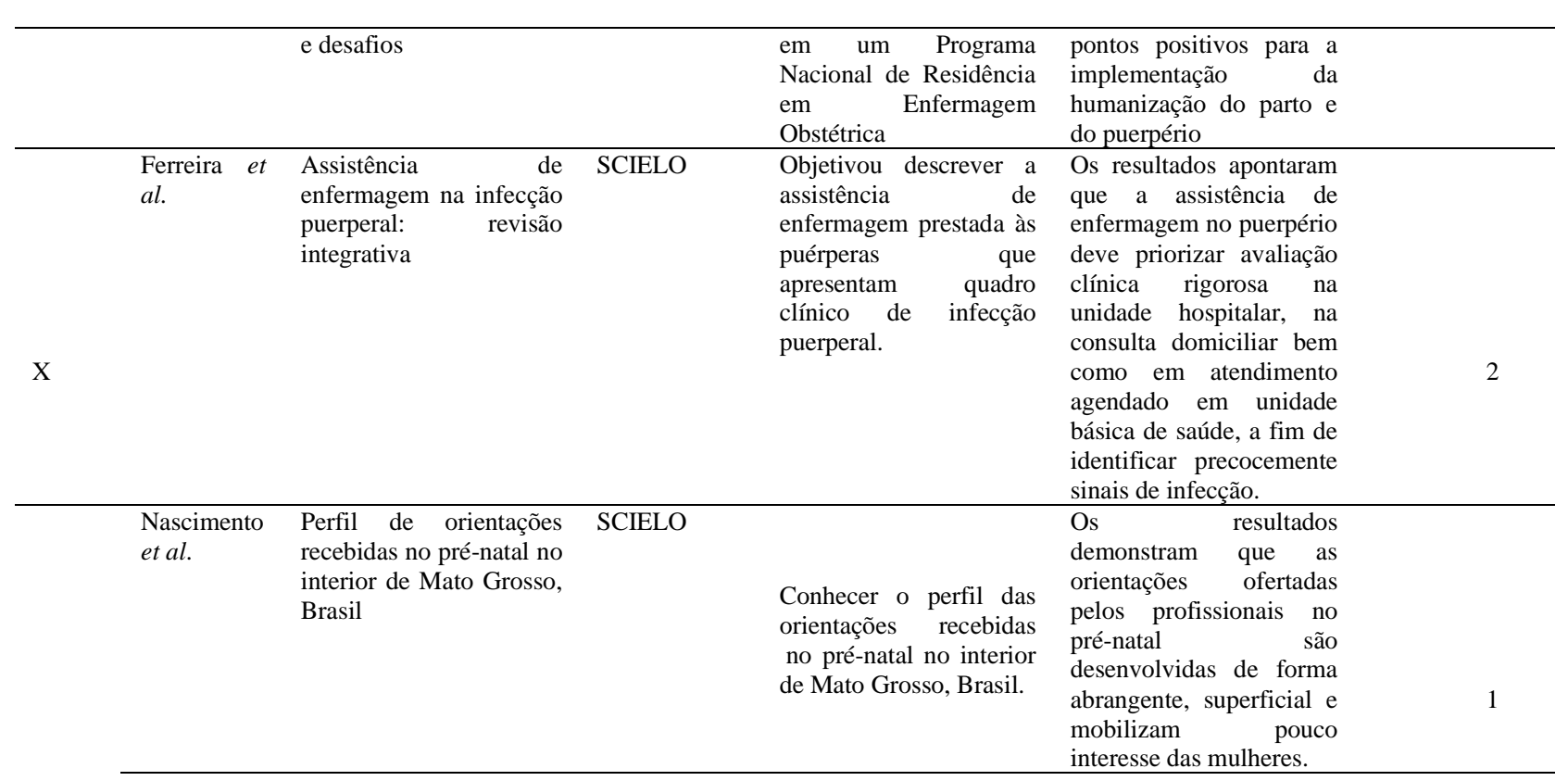

Fonte: Pesquisa direta na Biblioteca Virtual em Saúde (BVS) via Portal CAPES.

Os resultados encontrados na análise dos artigos, a partir do levantamento bibliográfico, propiciaram a formação de categorias temáticas para a melhor compreensão do tema aqui abordado e a discussão das principais informações discutidas na literatura científica sobre o Puerpério e os Cuidados de Enfermagem.

\subsection{Perfil das pacientes durante a transição puerperal}

Dentre as produções científicas analisadas, são descritos os perfis das puérperas em relação às características sociodemográficas e clínicas. Quanto às características sociodemográficas, são apresentadas: a causa da admissão. A respeito das características clínicas, são destacadas: condições de saúde segundo a teoria das NHB de Wanda Horta de Aguiar, aspectos multidimensionais da saúde.

Trata-se de um grupo de puérperas jovens, com nível de escolaridade que se concentra entre o ensino fundamental incompleto e médio completo, com baixa renda salarial mensal - que pode ser consequência da própria profissão/ocupação que usualmente está relacionada ao salário mínimo. A baixa escolaridade entre as mulheres no presente estudo retrata a situação educacional das jovens brasileiras que é considerada precária, em que aproximadamente 10\% das mulheres fazem curso superior. A renda mensal também revela um perfil familiar com pequeno poder aquisitivo, o que equivale ao perfil da maioria dos brasileiros.

No tocante a causa da admissão e ao tempo de permanência, Prior (2017) destacam que 44,2\% das puérperas pouca idade e falta de apoio e 34,9\% pela dificuldade de conviver com familiares. Ebling e Baratier (2020) relatam que 49\% das puérperas eram provenientes de domicílio próprio e 30\% moravam sozinhas e tinham dificuldades para se sustentarem sozinhas, sendo este o motivo principal da necessidade de apoio.

Em se tratando das produções científicas um dos temas mais abordados nos relatos são as alterações emocionais e físicas que ocorrem no período pós-parto. Em relação as questões emocionais as mulheres informaram que o puerpério foi um momento delicado, com muitos episódios de choro, desespero e até mesmo frustração.

No que diz respeito aos aspectos multidimensionais da saúde, algumas mulheres informaram que não tiveram dificuldades no puerpério e que passaram por este momento bem e tranquilas, assim como sem dificuldades na amamentação. Uma mulher relatou que fez o parto domiciliar e achou a experiência ótima pois já conseguiu dormir a primeira noite pós-parto em sua cama. 
Nos demais estudos foi observado que a família é outro ponto importante no puerpério, pode ser tida como a base deste momento, o alicerce e a força para superar os momentos mais difíceis. Assim como o apoio nos afazeres da casa, da rotina, com outros filhos e com o recém-nascido. Com estas situações, a Equipe de Enfermagem tem uma atuação necessária nos fatores que podem contribuir para um puerpério com complicações.

A Enfermagem acompanhou e orientou as mães antes do parto e após, seja na amamentação, nas mudanças físicas, emocionais e hormonais, planejando assim uma rede de apoio e cuidados voltados à estas complicações. Destaca-se então, que embora cada mulher possa apresentar diferenças no puerpério, todas receberam uma atenção especial, apoio e cuidado. As boas práticas foram realizadas pelo profissional de Enfermagem afim de amenizar as vivencias negativas no puerpério em todo os estudos desta categoria.

\subsection{Cuidados de Enfermagem à puérpera no contexto hospitalar}

A produção científica relacionada a essa temática vem a destacar as consequências dos cuidados de Enfermagem utilizados na avaliação para melhora da qualidade de vida da puérpera durante o pós parto e de que forma esses cuidados podem influenciar na qualidade de vida da puérpera durante esse período tão delicado.

Os dados dos estudos relacionados a esta categoria apontaram diferenças estatísticas em relação ao nível de satisfação das puérperas quanto ao número de gestações e tipo de parto. As mulheres com três ou mais gestações apresentaram maior nível de satisfação para o domínio educacional em relação às mulheres com duas gestações. Uma das possíveis interpretações para esses achados deve-se ao fato de que a experiência adquirida em gestações pregressas permitiu maior conhecimento da puérpera sobre as demandas de cuidado inerentes ao puerpério e aos cuidados com o recém-nascido.

Os estudos enfatizam que as mulheres que relataram satisfação com o trabalho de parto e nascimento foram aquelas que já tinham uma expectativa prévia sobre seu desempenho durante o parto e também participaram de atividades educativas que as ajudaram a esclarecer quanto ao que esperar desse momento. $\mathrm{O}$ foco do presente estudo foi avaliar a satisfação no puerpério imediato, que corresponde aos primeiros dez dias após o parto, havendo, portanto, a necessidade de estudos que avaliem o nível de satisfação da puérpera nas outras fases do puerpério.

Por outro lado, as mulheres que vivenciaram o parto vaginal relataram maior nível de satisfação comparado ao das mulheres de parto cesáreo, abrangendo tanto situações do domínio educacional como do domínio técnico-profissional. Uma das razões para esses achados pode estar relacionada ao próprio processo de recuperação da puérpera que passou por uma cesárea, procedimento associado a um maior nível de dor e limitação do autocuidado. Em um estudo com mulheres que vivenciaram o parto normal, a percepção do controle pessoal no momento do parto e nascimento foi um fator de satisfação para elas. Os dados sugerem que as puérperas de pós-cesárea têm outras necessidades, que não vem sendo contempladas adequadamente pela enfermagem.

A puérpera que confia e conhece a função do profissional que lhe presta assistência sabe o que pode esperar desse profissional e deposita nele suas expectativas quanto à sua recuperação. Estudos enfatizam que a melhor compreensão dos cuidados de enfermagem recebidos poderá levar a uma recuperação mais rápida no pós-parto. Os cuidados são baseados na comunicação e no desenvolvimento de objetivos comuns entre paciente e profissional e devem contemplar desde o alívio da dor até a relação de confiança entre eles - fatores que contribuem para gerar maior satisfação.

Desse modo, dentro do objetivo de proporcionar o bem-estar da puérpera, buscando alternativas para que sua recuperação no pós-parto ocorra da melhor maneira possível, todo cuidado de Enfermagem se baseia numa boa relação entre enfermagem e paciente, para que a primeira possa interferir prontamente sempre que houver necessidade. Os dados deste estudo sugerem que as demandas de cuidado da puérpera que se recupera de um parto cirúrgico sejam diferentes daquela que se recupera de um parto vaginal. Neste sentido, sugere-se uma reflexão quanto aos cuidados prestados à mulher que se 
submeteu a uma cesárea, com vias a satisfazer suas necessidades, mas sempre buscando contemplar os objetivos da unidade de saúde / hospitalar, que envolve o autocuidado e o cuidado ao recém-nascido.

\section{Conclusão}

A produção científica acerca dos cuidados de Enfermagem a puérpera destacou dois pontos importantes: conhecer o perfil dessas puérperas no tocante às suas características sociodemográficas e clínicas e a avaliação das mesmas. A partir dos dados discutidos neste estudo, conclui-se que conhecer, analisar e compreender as características das puérperas contribuiu para um aprofundamento na temática e uma reflexão dos principais pontos a serem avaliados quando se quer uma assistência sistematizada e individualiza, a fim de proporcionar melhor qualidade de vida durante o parto e pós parto.

O estudo se limita a produções científicas nacionais, muito embora este seja um ponto de suma importância para quem trabalha com a assistência a puérpera no Brasil, pois a realidade de cada país é particular e, portanto, deve ser analisada como tal. Recomenda-se estudos nessa temática, no que se refere às estratégias para a promoção da qualidade de vida dessas puérperas, pois já se sabe que conhecendo o perfil dessas puérperas, as estratégias podem ser construídas com base em evidências científicas.

\section{Referências}

Alpirez, L. A. et al. (2008) Validação de conteúdo de instrumento de avaliação do recém-nascido. Acta Paul Enferm., 31(2),123-9.

Andrade, C. J.; Baccelli, S, M. \& Benincasa, M. (2017) O vínculo mãe-bebê no período de puerpério: uma análise winnicottiana. Vínculo - Revista do NESME, 14(1), 1-13.

Araújo, A. M. S. (2016) Alterações emocionais (a) típicas no pós-parto: o relato das mães. Rev. Científica da Saúde, 2(1).

Brasil. (2006) Ministério da Saúde. Pré-natal e puerpério: atenção qualificada e humanizada - manual técnico. Área Técnica de Saúde da Mulher, Departamento de Ações Programáticas Estratégicas, Secretaria de Atenção à Saúde, Ministério da Saúde. (3a ed.).

Ministério da Saúde. (2012) Política Nacional de Atenção Básica. Departamento de Atenção Básica, Secretaria de Atenção à Saúde, Ministério da Saúde.

Ministério da Saúde. (2013) Secretaria de Atenção à Saúde. Departamento de Atenção Básica. Atenção ao pré-natal de baixo risco. Ministério da Saúde.

Ministério da Saúde. (2013) Atenção ao pré-natal de baixo risco. Secretaria de Atenção à Saúde. Departamento de Atenção Básica.

Castiglioni, C. M. et al. (2020) Práticas de cuidado no puerpério desenvolvidas por enfermeiras em Estratégias de Saúde da Família. Rev. Enferm. UFSM REUFSM, 10(50), 1-19.

Carvalho, V. L. et al. (2010) A influência da maternidade na vida da mulher contemporânea. Anais II - simpac, 1(1), 335-340.

Castro, A. C. O.; Duarte, E. D. \& Diniz, I. A. (2017) Intervenção do Enfermeiro às Crianças Atendidas no Ambulatório de Seguimento do Recém-Nascido de Risco. Revista de Enfermagem do Centro-Oeste Mineiro. 7(e1159), 1-9.

Corrêa, M. S. M. et al. (2017) Atenção no cuidado à saúde da mulher no puerpério. Cad. Saúde Pública, 33(3), e00136215.

Corrêa, M. S. M. et al. (2017) Acolhimento no cuidado à saúde da mulher no puerpério. Cad. Saúde Pública. 33(3).

Filho, A. C. A. A. et al. (2017) Aspectos epidemiológicos da mortalidade neonatal em capital do nordeste do Brasil. Rev Cuid. 8(3), $1767-76$.

Garcia, P. T. (2013) Saúde da Mulher Geral. Brasília: UNA-SUS-Universidade Aberta do SUS.

Gomes, G. F \& Santos, A. P. V. (2017) Assistência de enfermagem no puerpério. Revista Enfermagem Contemporânea. 6(2), 211-220

Krob, A. D. et al. (2017) Depressão na gestação e no pós-parto e a responsividade materna nesse contexto. Rev. Psicol. Saúde, 9(3), 3-16

Lucena, D. B. A. et al. (2018) Primeira semana saúde integral do recém-nascido: ações de enfermeiros da Estratégia Saúde da Família. Rev Gaúcha Enferm. 39(1), e2017-0068

Maciel, L. P. et al. (2019) Mental disorder in the puerperal period: risks and coping mechanisms for health promotion. J. res.: fundam. care. Online, 11(4), 1096-1102.

Maciel, L. P. et al. (2019) Transtorno mental no puerpério: riscos e mecanismos de enfrentamento para a promoção da saúde. Rev. pesqui. cuid. fundam. (Online), 11(4), 1096-102. 
Research, Society and Development, v. 10, n. 14, e574101422365, 2021

(CC BY 4.0) | ISSN 2525-3409 | DOI: http://dx.doi.org/10.33448/rsd-v10i14.22365

Pereira, T. R. C. et al. (2017) Existe associação entre os desconfortos no puerpério imediato e a via de parto? Um estudo observaciona. ABCS Health Sci. 42(2), 80-84.

Ruiz, M.T. et al. (2017) Perda hemática e sinais ou sintomas durante avaliação puerperal: implicações para a assistência de enfermagem. Rev enferm UERJ.

Silva, R. A. (2021) Gravidez em tempos de COVID-19: como a mudança dos protocolos de biossegurança afetam a mulher no momento do parto e no puerpério: revisão de literatura. Brazilian Journal of Health Review, 4(1),1356-1367.

Silva, L. P. et al. (2020) Assistência puerperal e a construção de um fluxograma para consulta de enfermagem. Rev. Bras. Saúde Mater. Infant., 20(1), 115127.

Sleutjes, F. C. M. et al. (2018) Fatores de risco de óbito neonatal em região do interior paulista, Brasil. Ciência \& Saúde Coletiva, 23(8),2713-2720.

Soler, D. R. et al. (2015) Qualidade de vida no puerpério: avaliação no pós-parto imediato, tardio e remoto. Revenferm UFPE.

Vilela, M. L. F \& Pereira, Q. L. C. (2018) Consulta puerperal: orientação sobre sua importância. Journal Health NPEPS. 3(1), 228-240.

WHO. (2015) World Health Organization. Estimates Developed by the UN Inter-agency Group for Child Mortality Estimation United. WHO 